\title{
The Promoter Targeting Sequence mediates epigenetically heritable transcription memory
}

\author{
Qing Lin, Qi Chen, Lan Lin, and Jumin Zhou' \\ The Wistar Institute, Philadelphia, Pennsylvania 19104, USA
}

\begin{abstract}
Large gene complexes frequently use "specialized" DNA elements to ensure proper regulation of gene activities. The Promoter Targeting Sequence (PTS) from the Abdominal-B locus of the Drosophila Bithorax complex overcomes an insulator, and facilitates, yet restricts, distant enhancers to a single promoter. We found that this promoter-targeting activity is independent of an enhancer's tissue or temporal specificity, and can be remembered in all somatic cells in the absence of promoter activation. It requires an insulator for its establishment, but can be maintained by the PTS in the absence of an insulator. More importantly, the promoter-targeting activity can be remembered after the transgene is translocated to new chromosomal locations. These results suggest that promoter targeting is established independent of enhancer activity, and is maintained epigenetically throughout development and subsequent generations.
\end{abstract}

[Keywords: Abdominal-B; PTS; insulator; promoter targeting; epigenetic inheritance; transcription memory]

Received June 9, 2004; revised version accepted August 27, 2004.

Cell identity, as specified by differential gene expression, relies on heritable epigenetic memory of gene activity through many cell cycles. The most notable examples are the functions of the Drosophila Polycomb Group (PcG) and Trithorax Group (trxG) gene products, which act through dedicated Polycomb/Trithorax Response Elements (PRE/TRE), located at numerous loci in the homeotic gene complex, to maintain the silenced or activated states of the local chromatin throughout development (Kennison 1995; Simon 1995; Hagstrom and Schedl 1997; Pirrotta 1998). However, most epigenetic chromatin marks acquired during development, and in the adult stages, affect only somatic cells and are not transmitted through the germ line. Any epigenetic alterations to the germ-line chromatin are thought to be erased during gametogenesis and fertilization to ensure totipotency in the gametes (Rakyan et al. 2001). However, there is growing evidence that specific marks on the chromatin or the DNA are transmittable through the germ line to the subsequent generations (Rakyan and Whitelaw 2003). The methylation-induced silencing of the agouti and axin-fused loci in mice (Morgan et al. 1999; Rakyan et al. 2003), the swi6-mediated silencing of the mating locus in yeast (Nakayama et al. 2000), and the transcription memory mediated by the Cellular Memory

${ }^{1}$ Corresponding author.

E-MAIL zhouj@wistar.upenn.edu; FAX (215) 898-0663.

Article and publication are at http://www.genesdev.org/cgi/doi/10.1101/ gad.1230004.
Module (CMM) (Cavalli and Paro 1998; Bantignies et al. 2003) near the Front-abdominal (Fab-7) region of the Drosophila Abdominal-B (Abd-B) locus, are just a few examples of known activities that can be epigenetically inherited through subsequent generations. These studies raise the possibility that germ-line chromatin is not entirely naive, and that certain regulatory mechanisms might be maintained in the germ-line chromatin and thus be transmittable to the next generation.

The homeotic gene complexes ANT-C and BX-C require elaborate regulatory controls to ensure that each homeotic gene is expressed at the appropriate level in a temporal- and tissue-specific manner. Each of these genes uses a very large and complex control region containing multiple "specialized" regulatory elements, in addition to tissue-specific enhancers. The $A b d-B$ locus is organized into multiple enhancer domains, termed infraabdominal (iab). Each $i a b$ regulates $A b d-B$ expression in a specific abdominal segment (see Fig. 1A) (Duncan 1987; Celniker et al. 1990; Mihaly et al. 1998). Each of these domains is functionally separated from the next by a domain boundary element, such as Frontabdominal-7 (Fab-7) or Fab-8 (Fig. 1A) (Gyurkovics et al. 1990; Galloni et al. 1993; Hagstrom et al. 1996; Zhou et al. 1996, 1999; Mihaly et al. 1997; Barges et al. 2000).

Boundary elements or insulators specifically block the activity of an enhancer when these elements are placed between the enhancer and a promoter. In their endogenous locations, insulators usually function as chromatin boundaries to prevent enhancers from ectopically ac- 

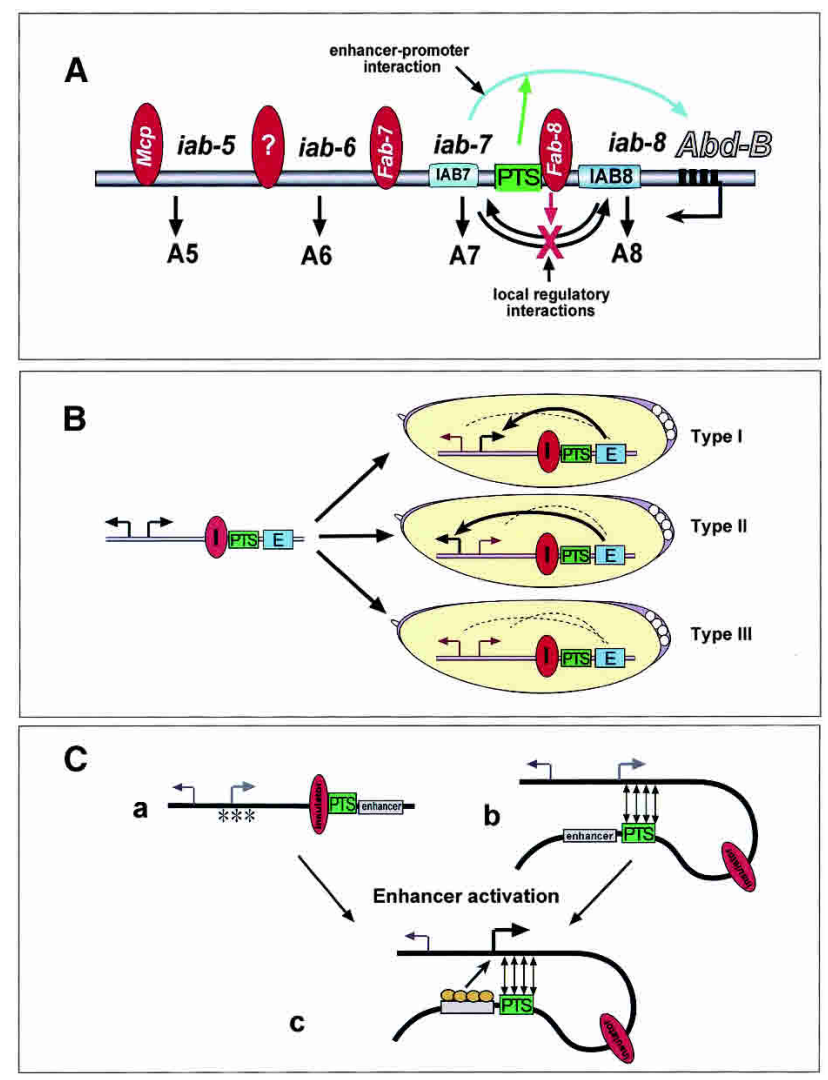

Figure 1. cis-regulatory interactions in $A b d-B .(A) A b d-B$ has an extended 3 ' regulatory region that can be subdivided into four regulatory domains, termed infra-abdominal (iab)-5, iab-6, $i a b-7$, and iab-8. Each of these controls the development of a corresponding abdominal parasegment (PS). For example, iab-5 regulates $A b d-B$ function in PS10 (roughly the fifth abdominal segment, or A5), iab-6 controls A6, and so on (Mihaly et al. 1998). Neighboring iabs are separated by domain boundary elements such as Fab-7 and Fab-8, which function to prevent regulatory interference between neighboring iabs. These elements are also potent insulators that can block enhancer-promoter interactions in transgenic constructs. The PTS element has an anti-insulator activity that allows an enhancer to activate a promoter despite an intervening insulator. It also facilitates long-range enhancer-promoter interactions and restricts the enhancer to a single promoter in the transgenic embryo. In the $A b d-B$ locus, PTS may mediate enhancer-promoter interactions by overcoming the intervening $F a b$ elements and facilitating the activities of the distal enhancers. (B) Three types of enhancer-promoter interaction in transgenic strains carrying the PTS element: Type I, the enhancer targets the proximal promoter; Type II, the distal promoter; Type III, no promoter targeting and the enhancer is presumably blocked by the insulator. (C) Potential mechanisms of epigenetic inheritance of promoter targeting. At least two models could be proposed. First, the transcription memory could be inherited by special chromatin or DNA modifications (part $a$ ) that are transmittable through the germ line so that in somatic cells, the PTS could re-establish promoter-targeting (part $c$ ). Second, the PTS could establish a stable chromatin loop between DNA near the enhancer and the promoter (part $b$ ). Such loops could survive meiosis, and be transmitted to the next generations (part $c$ ). tivating the wrong genes, and to restrict chromatin regulatory activities within defined domains by blocking the possible "spreading" of active or silenced chromatin along the DNA (Bell et al. 2001). This type of activity has been described with respect to histone acetylation in the chicken $\beta$-globin LCR (Prioleau et al. 1999), histone methylation in the yeast mating-type locus (Noma et al. 2001; Grewal and Elgin 2002; Hall et al. 2002), and long-range silencing of a Drosophila PRE (Sigrist and Pirrotta 1997; Mallin et al. 1998; Bell and Felsenfeld 1999). The best-studied insulators include the vertebrate ß-globin insulator (Chung et al. 1993; Bell et al. 1999), the H19/IGF-2 insulator (Bell and Felsenfeld 2000; Hark et al. 2000; Kanduri et al. 2000), the Drosophila scs, scs' elements (Kellum and Schedl 1992; Zhao et al. 1995; Gaszner et al. 1999), and the suppressor of Hairy wing $(\mathrm{suHW})$ insulator from the gypsy transposon (Geyer and Corces 1992; Dorsett 1993; Gerasimova et al. 2000).

The fact that both Fab-7 and Fab-8 possess potent enhancer blocking activity when assayed in transgenic flies raises the possibility that, within the $A b d-B$ locus, additional mechanisms must be in place to ensure that proper enhancer-promoter interactions are not blocked by this activity. A recent survey of a region $15 \mathrm{~kb}$ downstream from the $A b d-B$ promoter (Hendrickson and Sakonju 1995; Hopmann et al. 1995; Sipos et al. 1998) led to the identification of a novel cis-regulatory element, the Promoter Targeting Sequence (PTS; Fig. 1A) (Zhou and Levine 1999). The minimal 290-bp PTS exhibits an anti-insulator activity, facilitates long-range enhancer-promoter interactions, and usually restricts the enhancer activity to a single promoter when more than one is present in the same transgene (Lin et al. 2003). These unique properties of the PTS suggest that it may normally regulate highly specific, long-range enhancer-promoter communications in the $A b d-B$ locus by overcoming the enhancer-blocking activity of the Fab-8 element (Fig. 1A) (Zhou and Levine 1999).

Three types of transgenic strains are usually obtained when transgenes carrying the PTS (and an insulator) are introduced into the fly genome by $P$-element-mediated transformation. In Type I, the enhancer targets the proximal promoter; in Type II, it targets the distal promoter; and in Type III, no promoter targeting occurs, and the enhancer remains blocked by the insulator (Fig. 1B). Although the promoter-targeting function varies from strain to strain, this effect is stable in subsequent generations within a specific strain. This phenomenon has led us to hypothesize that promoter targeting is epigenetically heritable. In this study, we report that the PTS cotargets multiple enhancers (i.e., targets two or more enhancers that are divergent in tissue specificity and temporal patterns) to the same promoter. We also found promoter targeting requires an insulator to be established but could be maintained in all successive generations by the PTS in the absence of the insulator. Finally, we present evidence that promoter targeting is stable after $P$-element transposition to new chromosomal locations. These results suggest that the PTS maintains an 
epigenetic transcriptional memory that can be transmitted to successive generations.

\section{Results}

The PTS targets multiple enhancers to the same promoter

In principle, promoter targeting could be affected by several factors such as the local chromatin structure, the identity of the enhancer or the promoter, and the arrangement of different cis elements within the transgene. Among these, the enhancer could play an important role. For example, the PTS could selectively sense the weak, insulator-attenuated enhancer-promoter interaction, and reinforce it on a specific promoter. This model predicts that different enhancers could be targeted independently to different promoters in the same transgenic strain. Alternatively, a targeted promoter could be determined independent of enhancer identity or enhancer activity. This model suggests that the PTS could target all nearby enhancers to the same promoter regardless of their tissue or temporal specificity.

To distinguish between these two models, we challenged the PTS with three different enhancers in the same transgene: an embryonic Neural Ectoderm Enhancer (NEE) from the rhomboid gene (Ip et al. 1992), an embryonic IAB5 enhancer from the $A b d-B$ locus (Busturia and Bienz 1993), and an adult eye-specific enhancer glass from the glass gene (Moses and Rubin 1991) (see Fig. 2). The transgenic vector (W191; Fig. 2I) carrying these enhancers also contains two divergently transcribed genes, white $(w)$ and eve-GFP (Small et al. 1993). In the absence of the insulator, suHW, and the PTS, each of these enhancers is able to activate both $w$ and eve promoters (data not shown), but in the presence of both suHw and the PTS, we obtained three types of transgenic strains as illustrated in Figure 1B. From 31 strains analyzed, different transgenic enhancers target $W$ in four strains and GFP in three other strains. In each of these targeted strains, both NEE and IAB5 target the same promoter, a phenomenon we refer to as cotargeting. An example of a Type I, proximal eve promoter-targeted strain, is shown in Figure 2A-D, and a Type II, distal $w$ promoter-targeted strain is shown in Figure 2E-H. In a separate experiment, we tested hairy stripe I (HI) (Riddihough and Ish-Horowicz 1991) and IAB5 in transgenic vector W199 (see summary in Fig. 2J). Cotargeting of these two enhancers was also observed. Because NEE, HI, and IAB5 enhancers use different activators and repressors, these data suggest that promoter targeting is independent of enhancer identity or enhancer-interacting proteins.

To test whether the glass enhancer is also cotargeted with the embryonic enhancers, we surveyed the eye color of adult flies for $W$-targeted (Type II) versus nontargeted (Type III) or GFP-targeted (Type I) strains. We found that $w$-targeted strains have consistently red or bright red colored eyes, whereas the remaining strains usually have yellow to orange eyes. For example, the eyes of $w$-targeted W191 are bright red in all four cases
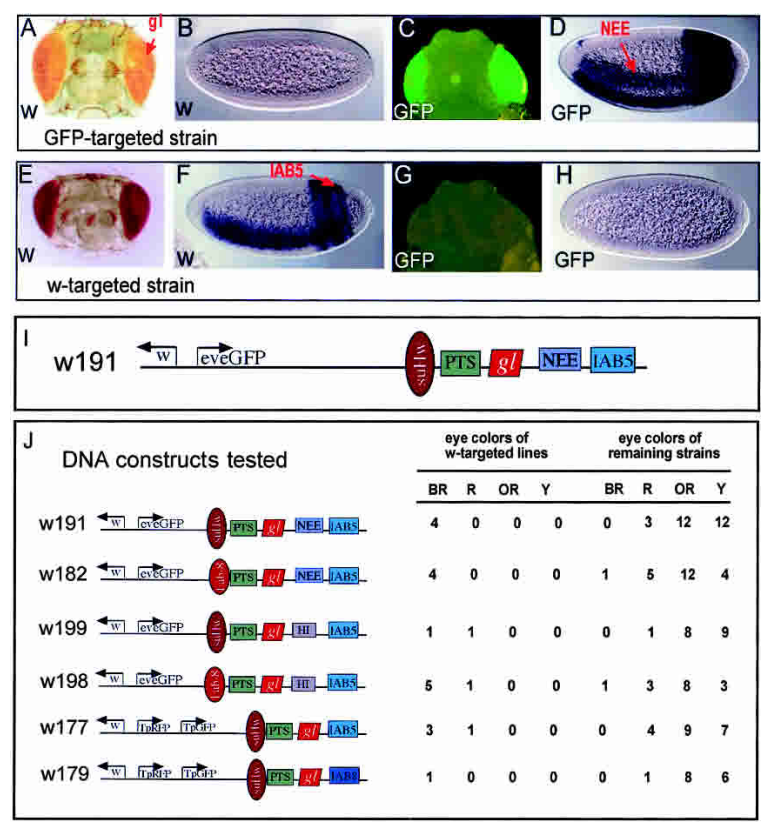

Figure 2. The PTS cotargets multiple enhancers to the same promoter. $(A-H)$ Transgenic animals carry the transgene W191 that contains three enhancers: the embryonic NEE, embryonic IAB5, and the adult, eye-specific glass enhancer. The insulator suHw and the PTS were inserted between the 3' of GFP and the enhancers. The activities of the three enhancers are shown in either $w$ - or GFP-targeted lines. Blastoderm-stage embryos (from 2-4 h old) were collected, fixed, and subjected to in situ hybridization using anti-sense RNA to either $w$ or GFP as probes. Processed embryos were mounted on slides and photographed. All embryos shown in this study are parasagittal shots with anterior pointing left and dorsal side up. (A) A typical orange eye color for a GFP-targeted line, W191-21, showing basal level of $W$ transcription. $(B)$ Neither NEE nor IAB5 activates $W$ in this strain. $(C)$ Glass activates GFP in the eye. $(D)$ Both NEE and IAB5 enhancers strongly activate the GFP gene. $(E)$ Strong $w$ activation (bright red eye) in the adult eye is shown in a $\mathrm{W}$ targeted line, W191-17. $(F)$ Both of the embryonic enhancers activate $w$ in the embryo. $(G, H)$ No GFP activation is detected in the eye or the embryo. (I) Transgenic DNA construct W191 providing results in $A-H$. (J) A summary of eye color distribution from six different transgenes that contain multiple enhancers that can be targeted by the PTS. (BR) Bright red; (R) red; (OR) orange; $(\mathrm{Y})$ yellow.

(Fig. 2E), whereas the eyes in nontargeted strains and GFP-targeted strains have yellow or orange, but only in a few cases, red eyes (Fig. 2A,J). These consistently high levels of $W$ activation in adult eye (evidenced by bright red eye color) when embryonic enhancers are targeted to $W$ are seen in all six transgenes tested (summarized in Fig. 2J). In the absence of glass, the eye color is not affected by which promoter is targeted (data not shown). When GFP expression is examined in pupa stage (where GFP is not masked by $w$ expression), GFP-targeted strains display strong green fluorescence whereas nonGFP-targeted lines show almost no GFP activity (Fig. 2, cf. C and G). These results suggest that glass is also targeted to the same promoters as the embryonic enhanc- 
Lin et al.

ers, NEE, HI, and IAB5. Thus, the cotargeting of these enhancers (both embryonic and adult) strongly supports the epigenetic maintenance model.

The PTS functions only in the presence of an insulator in transgenic embryos

In addition to overcoming an insulator, the PTS also facilitates the distal enhancer and restricts it to a single promoter, indicating that the PTS does not simply cancel the function of an insulator (Lin et al. 2003). For this reason, we examined whether the PTS exhibits these additional activities in the absence of an insulator. We constructed four types of synthetic $P$-transposons to test this hypothesis. The first (Table 1A) contains two divergently transcribed reporter genes, $W$ and Transposase $(T p)-1 a c Z$, as well as one of the lac $Z 3^{\prime}$-located enhancers such as IAB5, IAB8, or NEE. The second type (Table 1B) also contains the insulator, $s u H W$, inserted between the $3^{\prime}$ end of lacZ and one of the enhancers. The third (Table 1C) includes only the PTS in this position, whereas the last type (Table 1D) contains both an insulator ( $s u H w$ or
Fab-8) and the PTS between the 3 '-end of $l a c Z$ and one of the downstream enhancers. Individual transgenic strains for each of these constructs were analyzed, and the results are summarized in Table 1 . In the absence of the insulator or the PTS, each of the enhancers tested could activate both $W$ and lacZ (Table $1 \mathrm{~A}$ ), whereas when an insulator is inserted between the 3 '-end of lac $Z$ and the enhancer, transcription of both $w$ and lac $Z$ is blocked (Table 1B). In contrast, inserting only the PTS did not affect enhancer-promoter interactions in any of the tested transgenes (Table 1C). Promoter targeting is observed only when both an insulator and the PTS are inserted in the transgene (Table 1D). The relative order of the insulator and the PTS does not affect promoter targeting (cf. W87 and W88, or W97 and W98 in Table 1D).

A specific example of insulator requirement for promoter targeting is shown in Figure 3: When IAB5 is placed at the $3^{\prime}$-end of $1 a c Z$, it activates both the divergently transcribed $w$ and $T p-l a c Z$ fusion genes in the posterior region of the early embryo (see W91 in Fig. 3A). This activity is greatly reduced by inserting the $s u H W$ insulator (see W71 in Fig. 3C). In contrast, the addition of

Table 1. Summary of staining results of different transgenic constructs

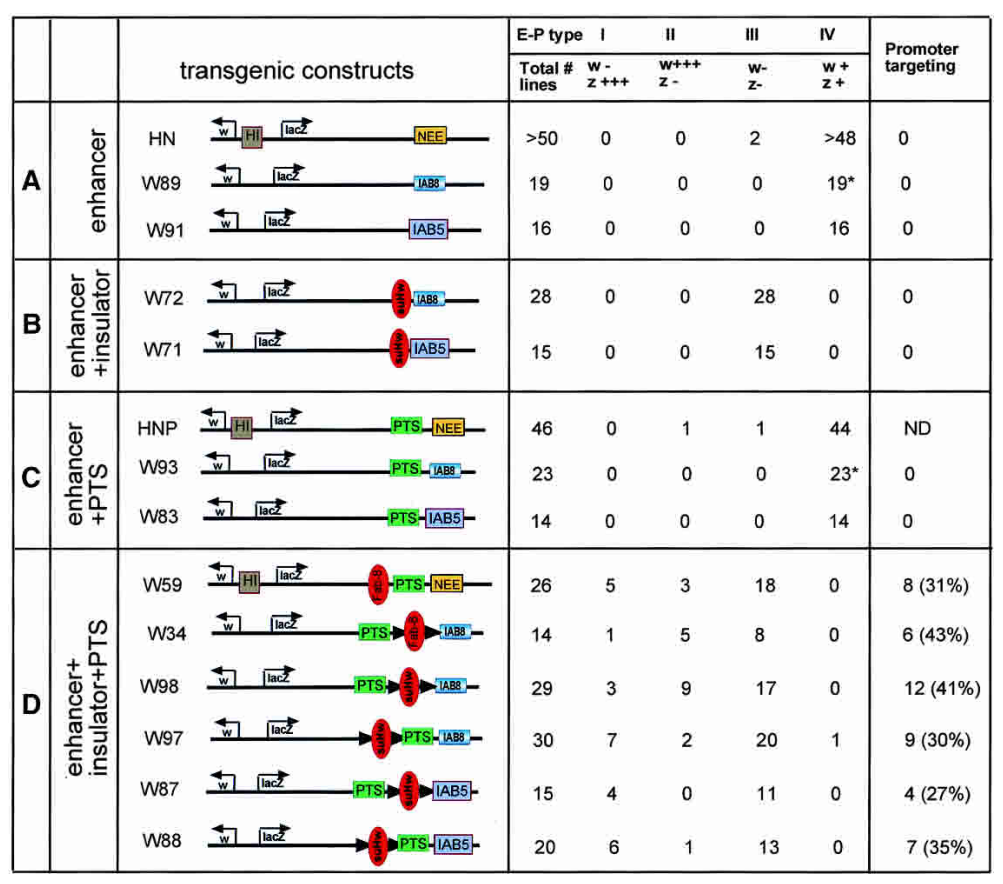

The constructs are classified into four groups: (I) enhancer alone inserted at the $3^{\prime}$-end of Tp-lacZ (A); (II) enhancer plus an insulator located between the enhancer and the Tp promoter $(\mathrm{B})$; (III) enhancer plus the PTS (C); and (IV) enhancer plus the PTS and insulator inserted between the enhancer and the Tp promoter (D). Three enhancers, NEE, IAB5, and IAB8 were analyzed and two insulators, Fab-8 and suHW, were tested. With the exception of constructs HN, HNP, and W59, which contain a second HI enhancer as a control, all constructs carry a single enhancer. Enhancer- $W$ and enhancer-lac $Z$ interactions are grouped into four categories based on staining patterns: Type I, no activation of $w$ but strong activation of lac $Z$ shown as $w-z+++$; Type II, strong activation of $w$ but no activation of lacZ shown as w+++z-; Type III, no activation of either $w$ or lacZ shown as w-z-; and Type IV, activation of both $w$ and lacZ with low level of transcription shown as $\mathrm{w}+\mathrm{Z}+$.

${ }^{\star}$ Indicates that enhancer-lac $Z$ interaction is stronger than that of enhancer- $W$ interaction. Promoter targeting indicates the fact that the lacZ 3 '-enhancer selectively activates only one of the divergently transcribed $w$ or Tp promoters (type I or II) and its activity is much stronger than that of the enhancer alone when placed at the same position. 

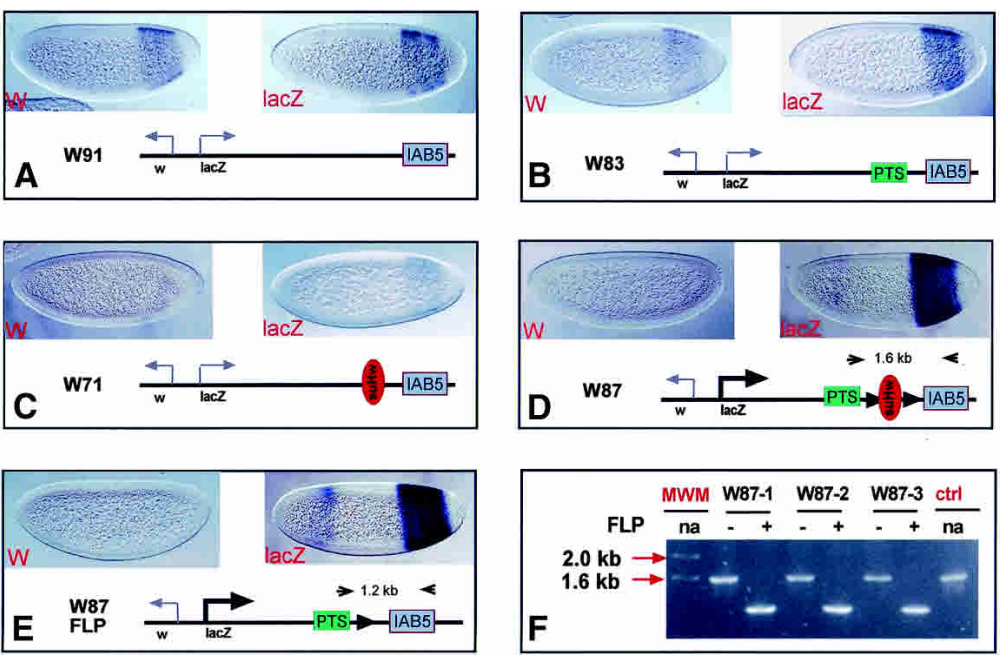

Figure 3. The $s u H w$ insulator is initially required for promoter targeting of the PTS. This transgene contains two divergently transcribed $W$ and Transposase (Tp)-lac Z fusion genes. A 1-kb IAB5 enhancer was inserted at the $3^{\prime}$-end of the lac $Z$ gene. Between the $3^{\prime}$-end of $l a c Z$ and the IAB5 enhancer, the PTS, the $s u H w$ insulator, or both were inserted. (A) IAB5 activates both $W$ and $l a c Z$ when placed downstream from lacZ. $(B)$ The PTS alone does not alter IAB5- $W$ or IAB5-Tp interaction. (C) The $s u H w$ insulator blocks the IAB5 enhancer. Very little or no transcription could be detected from the lac $Z$ or the $w$ gene. $(D)$ When both suHw and PTS are inserted, the PTS overcomes the insulator and targets IAB5 to one of the 5 '-promoters. IAB5 targets lacZ in four out of 15 strains examined. An example of a lac $Z$-targeted line (W87-1) is shown. (E) Deletion of the insulator by FLP-FRT-mediated recombination from the integrated transgene does not affect promoter targeting. IAB5 continues to activate lacZ exclusively. $(F)$ Genomic PCR of three representative lines demonstrated that the insulator was, indeed, deleted from the transgene. Note the 400-bp reduction of the PCR fragment size after the deletion of the suHw insulator.

the 625-bp PTS alone between the 3 '-end of lacZ and the IAB5 does not alter the strength or the patterns of IAB5-W or IAB5-Tp interactions significantly (Fig. 3, cf. A and B). However, when the 625-bp PTS and the suHw insulator are inserted together in the same position (see W87 in Fig. 3D), the PTS directs distinct anti-insulator and promoter-targeting activities. A strain displaying a selective IAB5-Tp interaction is shown in Figure $3 \mathrm{D}$, W87. The IAB5 activity is noticeably stronger than that of either Figure $3 \mathrm{~A}$ or B, confirming that the PTS also facilitates enhancer-promoter interactions. From a total of 15 strains examined, four exhibit the staining pattern shown in Figure 3D, and the rest display no promoter activation. Presumably, the PTS does not function in these strains, and IAB5 is blocked by the suHw insulator (see Table 1D). These results clearly indicate that an insulator is required for the promoter-targeting function of the PTS.

\section{The PTS maintains promoter targeting} after the deletion or the inactivation of an insulator

Our previous studies indicated that the PTS is necessary for both the initiation and the maintenance of promoter targeting (Zhou and Levine 1999; Lin et al. 2003). Here we have just presented evidence that an insulator is required for the initiation of promoter targeting. To determine whether it is also needed to maintain promoter targeting, we deleted the insulator from targeted strains using the FLP-FRT technique (Golic and Lindquist 1989), which permits the assessment of the test DNA in the same chromosomal position, thereby controlling for position effects. The $P$-element transformation vector W87 contains a pair of Flipase recognition sites, FRT, flanking the suHw insulator. We mated W87 transgenic lines (shown in Fig. 3D) with Flipase-expressing flies $\mathrm{X} 7$ to remove the $s u H w$ insulator from the integrated $P$-elements, and analyzed the offspring. When these embryos were collected and hybridized with $w$ and lac $Z$ probes, in parallel with the nonflipped strains, no difference in staining strength or staining pattern could be seen (Fig. 3, cf. D and E). Similar results were obtained when $w$-targeted strains were analyzed (data not shown). To ensure that the recombination, indeed, removed the suHw insulator, genomic PCR using primers against the suHw flanking region in the transgene was performed. As shown in Figure 3F, each of the three strains analyzed showed an expected 400-bp reduction in the size of the PCR fragments after the introduction of FLP recombinase, indicating that the suHw insulator was removed from the integrated transgenes. These results strongly suggest that the promoter-targeting function of the PTS can be maintained in the absence of an insulator in subsequent generations. A similar result was obtained when the insulator deletion analysis was done to transgenic strains carrying W98 (Fig. 5A-E) and W97 (data not shown).

Instead of deleting the insulator, we also inactivated the suHW insulator by introducing the transgene W98 into a genetic background mutant for the insulator protein Mod (mdg4) (Dorsett 1993; Cai and Levine 1995; Gerasimova et al. 1995; Scott and Geyer 1995). Although the insulator function is lost in the control W72 transgene in $\bmod (\operatorname{mdg} 4)$ mutant embryos, we did not observe any loss of promoter-targeting function in W98 in the same genetic background (data not shown), indicating the loss of insulator activity did not impede promoter targeting. To test whether the result seen with $s u H w$ is a general property of insulators, we also deleted the Fab-8 insulator from promoter-targeted strains carrying the W34 transgene (Table 1, group IV). None of the examined strains showed any loss of promoter targeting as a result of the insulator deletion (data not shown). Finally, we followed several of the insulator-deleted promoter targeted strains for $\sim 20$ generations, and did not detect any loss of promoter targeting. Taken together, 
these results clearly suggest that although an insulator is initially required, it is dispensable once promoter targeting is established, and that the memory of promoter targeting is stable through successive generations.

\section{Promoter-targeting memory could survive transgene transposition to new chromosomal locations}

Two possible hypotheses may explain why an insulator is required only initially for promoter targeting, and can be subsequently removed from the chromosomally integrated transgene in promoter-targeted strains. First, an insulator may be necessary to direct the transgene to a chromosomal location where promoter targeting could occur, so that once the transgene is integrated the insulator is no longer needed. Alternatively, when promoter targeting is established, an insulator-independent, epigenetically heritable memory is created to ensure promoter targeting in successive generations. We reasoned that if promoter targeting is epigenetically heritable through the germ line (as opposed to being stable from one cell cycle to the next), it might be possible for this memory to be carried with the transgene when it is moved to new chromosomal locations by introducing transposase into the germ-line lineage. For this reason, we mobilized several PTS transgenic lines, and examined the effects of new chromosomal insertion sites on promoter targeting.

First, as a control, we mobilized nontargeted strains from three similarly constructed transgenes, W14, J18, and W123 (Table 2A). All three contain the leftwardtranscribed $W$ and rightward-transcribed $T p-l a c Z$ gene, the lacZ 3'-located IAB8 enhancer, and the suHw insulator between the enhancer and the promoters, as shown in Table 2. In addition, W14 contains the 290-bp PTS located between the insulator and IAB8 (similar to W97 in Table 1), J18 contains the 625-bp PTS and a 3-kb spacer from the $\lambda$ genome separating the PTS from the suHw insulator, whereas W123 contains the 625-bp PTS and a 300-bp NEE enhancer located between the $W$ and $T p$ promoters. Transgenic strains carrying each of these constructs were mated with a strain expressing the $\Delta 2,3$ transposase. The progeny from these crosses carrying both a $P$-element and the transposase were mated with yw flies to establish stocks for analysis in embryos by in situ hybridization using antisense RNA probes against the $w$ or lacZ genes. When each of the nontargeted strains was transposed to new locations, we could readily recover promoter-targeted lines (Table $2 \mathrm{~A})$. We generated 12 strains from W14-1: Three targeted the Tp promoter (Type I), four targeted $w$ (Type II), and five showed no promoter targeting (Type III). Of 16 new insertion strains from J18-1, two displayed Type I, three exhibited Type II, and 11 showed no targeting. Similarly, of 12 W123-27 lines analyzed, two showed Type I, four showed Type II, and six exhibited no targeting. These results indicate that promoter-targeting strains can be obtained by $P$-element transposition of a nontargeted line, suggesting that the chromatin structure on a nontargeted $P$-element does not prevent promoter targeting upon integration into new chromosomal locations.
We then mobilized promoter-targeted strains carrying W32, W59, and W123. W32 contains a 5.3-kb DNA including the PTS, Fab-8, and IAB8, inserted at the 3 '-end of lacZ. W59 contains the PTS, Fab-8, and NEE in the same position. In addition, W59 contains a reference enhancer, HI, placed at the 5 '-end of lacZ (see Table 1; Fig. 4). W123 contains NEE at the promoter-proximal position and suHw, PTS, and IAB8 at the 3 '-end of lacZ (Table 2). We mobilized three $W$ promoter-targeted strains, W32-1, W59-1, and W123-14. When new insertion strains were examined, all exhibited the same pattern of promoter targeting to the $w$ promoter; no lacZtargeted strains were recovered (Table $2 B$ ). In situ hybridizations to the $W$ and $1 a c Z$ transgenes for W59 are shown in Figure 4. In $w$-targeted strains, the $\mathrm{HI}$ and the distal NEE enhancers are mutually inhibitory in the anterior region where both enhancers are active (see Fig. 4A; detailed studies to be described elsewhere). This activity allows us to test whether the new insertion strains have lost promoter targeting even when the original $P$-element is still present, because a nontargeted strain would produce strong $W$ activation by $\mathrm{HI}$ in the anterior region (Fig. 4B). As a result, when both the starting $w$-targeted $P$-element and a new nontargeted $P$-insertion exist in the same strain, a composite staining of both transgenes will be observed, and the inhibition of transcription in the anterior region will be masked by the strong HI-W interaction from the nontargeted strain. From a total of 17 new insertions analyzed, no reactivation of $\mathrm{HI}$ was observed. A typical new insertion is shown in Figure 4C, whereas a new transposition, W59-1 $\Delta 10$ from the second to the third chromosome, is shown in Figure 4D. In either case, there was no reappearance of robust $\mathrm{HI}-W$ interaction similar to that in Figure 4B (indicating a lost of promoter targeting) or NEE-lacZ interaction (indicating targeting to a different promoter), suggesting that there is no loss of promoter targeting or switching of targeted promoter.

We next tested whether the insulator is required for generating heritable promoter targeting at new insertion sites. We transposed promoter-targeted strains carrying W98 and W97 after the insulator had been deleted from the integrated transgenes (summarized in Table 2C). As shown in Figure 5C, in the original insertion line (W9819), the IAB8 enhancer was targeted to the Tp promoter in the presence of $s u H w$ and the PTS. This is evident as the enhancer strongly activates the $T p$ promoter as compared with IAB8 alone (W76 in Fig. 5A), and the activity is restricted to the Tp promoter. The deletion of $s u H W$ from this strain did not affect the PTS-mediated promoter targeting. The insulator-deleted strain was then mobilized, and a total of nine derivative strains were obtained that lack the original $P$-element as determined by inverse PCR. When the expression of $w$ and $1 a c Z$ were analyzed, seven of nine strains exhibited strong IAB8$T p-l a c Z$ interaction but no IAB8-W interaction, a pattern indistinguishable from the starting promoter-targeted strain (Fig. 5, cf. D and F). In the remaining two, one had lost promoter targeting, and the other had no apparent promoter activity. We also analyzed a $W$-tar- 
Table 2. Summary of enhancer-promoter interactions after P-element transposition

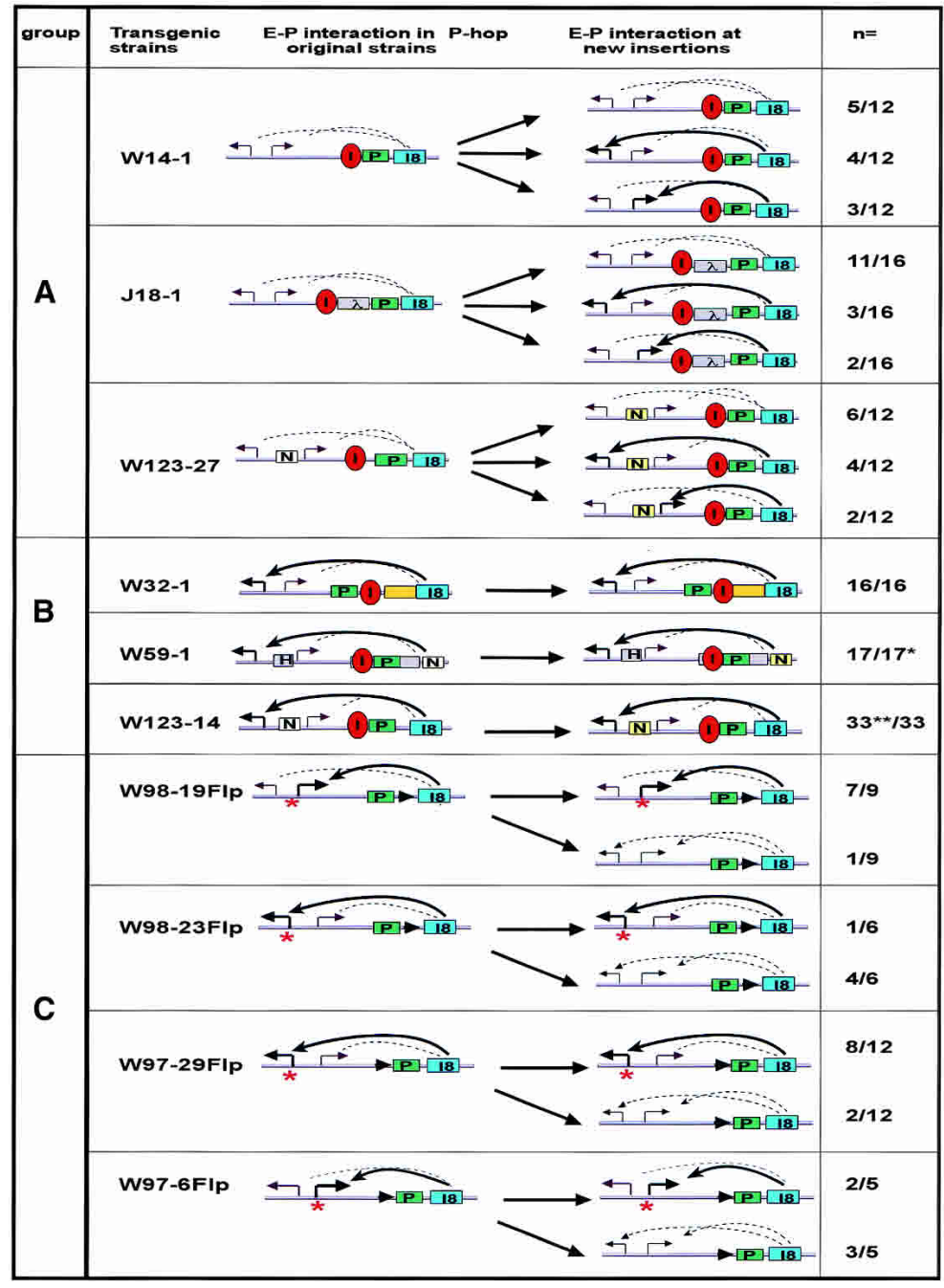

Red oval represents insulator; green rectangle represents the PTS; and N, I8, and H represent NEE, IAB8, and H1 enhancers, respectively. (A) Three representative lines carrying W14, J18, and W123 were transposed to new locations. Each of the three produced promoter-targeted strains. (B) Promoter targeting after $P$-element transposition of targeted strains. (C) Summary of promoter-targeted versus non-promoter-targeted strains obtained from transposing in insulator-deleted, promoter-targeted strains (W98-19/W98-23Flp, W97-29/W97-6; see Fig. 4). An inverse PCR screen was conducted to select strains that have lost the original $P$-element.

${ }^{\star}$ Indicates that two of the W59-1 lines are transposed from the second to the third chromosome.

${ }^{\star \star}$ Two of the 33 new insertion strains obtained from W123-14 are transposed from the second to the third chromosome.

geted, W98-23, for promoter targeting after transposition. A total of six lines were isolated, one of which remained targeted to the $w$ promoter, but four had lost promoter targeting, resulting in the weak activation of both $w$ and lacZ by IAB8. The remaining strain produced no transcription from either promoter.

W97 was constructed similar to W98 except the position of the insulator and the PTS was switched (Table 1D). As can be seen from the summary in Table 1D, the PTS mediates robust promoter targeting from this position. We first analyzed a $w$-targeted strain, W97-29: The deletion of suHw insulator did not affect promoter targeting to the $W$ promoter (data not shown). After transgene mobilization, 12 new insertion strains were recov- ered, eight of which retained promoter targeting, two of which lost promoter targeting, and the remaining two exhibited no detectable transgene expression (summarized in Table 2C). The transposition of a lacZ-targeted strain, W97-6, generated five strains, two retained promoter targeting, and one lost promoter targeting.

These results indicate that promoter targeting is remembered to a significant degree during $P$-transposition in the absence of an insulator, suggesting the insulator is not required in maintaining promoter targeting during this process. It remains possible, however, that insulator may increase the percentage of inheritance in transposed strains. These results are in contrast to that from obtaining promoter-targeted strains by the standard injection 
Lin et al.
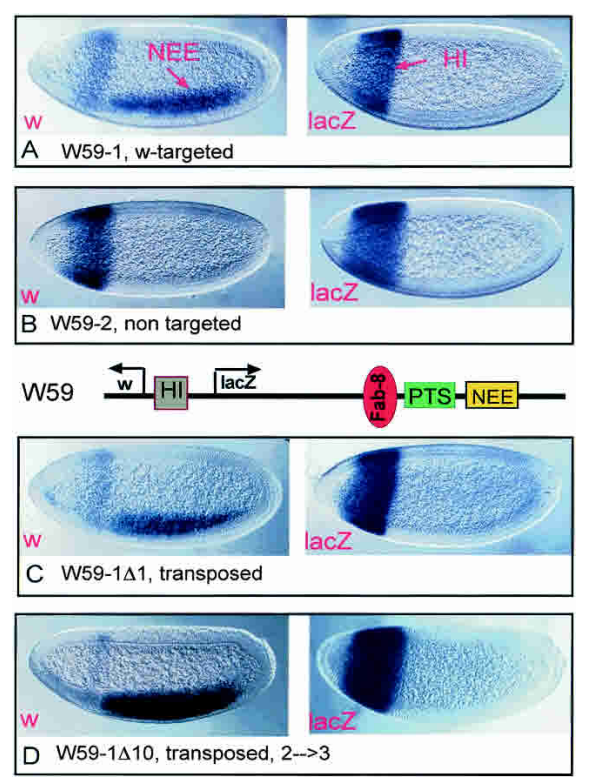

Figure 4. Promoter targeting is memorized after $P$-element transposition. (A) $W$ promoter targeting of the NEE enhancer in W59-1 strain. NEE activates $w$ exclusively; no NEE-lac $Z$ interaction could be detected. Note that the anterior third of the NEE enhancer is repressed, and the reference enhancer $\mathrm{HI}$ is also inhibited. (B) Promoter activity of a nontargeted strain (W59-2) showing robust $\mathrm{HI}$ enhancer function on both $W$ and Tp promoter, but no transcription activation by NEE. $(C)$ Enhancerpromoter interactions of a strain (W59-1 $\Delta 1$ ) obtained by transposing the original W59-1 strain. $(D)$ Transgene transcription of a strain (W5901 $\Delta 10)$ that was transposed from the second to the third chromosome.

(Table 1C), or by transposing nontargeted strains where an insulator is required (data not shown). Clearly, the history of promoter targeting could allow the insulatordeleted transgene to bypass the insulator requirement for promoter targeting when it is inserted into new chromosomal locations after transposition. Thus, these results suggest that a memory of the targeted promoter in the original strain could be transmitted through the germ line.

\section{Discussion}

We have presented several lines of evidence suggesting that the PTS-mediated promoter-targeting function is epigenetically heritable in both somatic and germ-line cells. First, we showed that promoter targeting is independent of tissue or temporal specificity of enhancers, and is memorized in the absence of transcription activity throughout development. Second, we demonstrated that promoter targeting requires an insulator for establishment but can be maintained by the PTS alone in all successive generations. Third, we observed that promoter-targeting memory is stable even after a transgene is translocated to new chromosomal locations.

\section{Enhancer cotargeting}

When given the choice of two promoters in the transgene, the PTS usually targets just one, resulting in exclusive transcription activation of the targeted promoter. What determines which promoter is targeted in a specific transgenic strain is not known, but two models could be proposed regarding the role of the enhancer in promoter selection. First, promoter targeting is determined partially by the enhancer or transcription factors bound to the enhancer. Second, it is epigenetically determined and maintained independent of the identity of the enhancer, and the enhancer-binding proteins. The first model predicts that if multiple enhancers exist in the transgene, the PTS could target different enhancers independently. For example, one enhancer could be targeted to the proximal Tp promoter, whereas the other could be targeted to the distal $w$ promoter in the same transgenic strain. Our results shown in Figure 2 demonstrated that this is not the case. In all transgenic strains examined, different enhancers are cotargeted to the same promoter. These results strongly support the epigenetic inheritance model. Consistent with this model, we observed that both adult eye enhancer glass and embryonic enhancers IAB5 are cotargeted to the same promoter (Fig. 2). Because the adult glass enhancer and the embryonic enhancer IAB5 are active in different tissues and at different times during the fly life cycle, cotargeting of these two enhancers suggests that in every somatic cell during the life span of the fly, the PTS maintains a memory of the target promoter should an enhancer become active during development.

\section{Mechanisms and implications of insulator requirement in promoter targeting}

Several models could be proposed to account for the role of the insulator in PTS function: First, an insulator may be necessary to direct the transgene to "special" nuclear compartments that are compatible with PTS function. The suHw insulator is known to cause the transgene to associate with the nuclear envelope /Gerasimova et al. 2000), whereas the vertebrate $\beta$-globin HS4 insulator has been shown to associate with the nucleolus (Yusufzai et al. 2004). In addition, artificially attaching transgenes to nuclear pores creates a chromatin boundary in the transgene (Gerasimova et al. 2000; Ishii et al. 2002). Although it provides an interesting mechanistic explanation, this model could not explain why the insulator is dispensable once promoter targeting is established, because the loss of insulator would dislodge the transgene from the nuclear compartment required for PTS function. The second possibility is that insulator DNA may cause the $P$ element to integrate into special regions of the chromosome during $P$-mediated transformation where the PTS can interact with the appropriate local chromatin structure favorable for promoter targeting. Although specific regulatory DNA sequences have been observed that cause preferential insertions into specific chromosomal locations (Bender and Hudson 2000; Kassis 2002), such a 

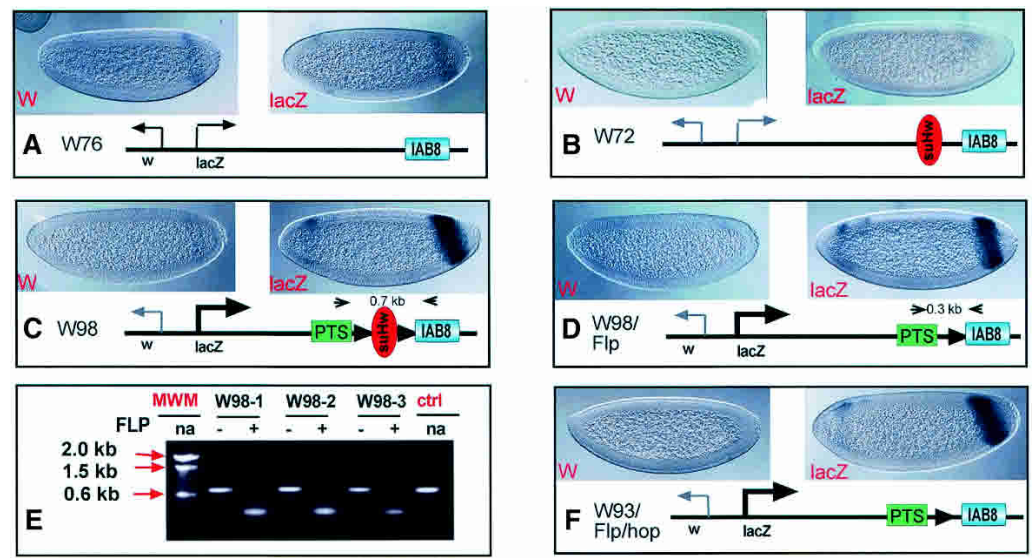

Figure 5. Effects of insulator deletion and transgene mobilization on the promoter targeting of the IAB8 enhancer. (A) The activities of the IAB8 enhancer on the $W$ and $T p$ promoter when it is located at the 3 '-end of $1 a c Z \sim 4.5$ and $5.1 \mathrm{~kb}$ away from the $T p$ and $w$ promoter, respectively. $(B)$ The $s u H W$ insulator blocks the IAB8 enhancer activity, no transcription can be detected from either of the promoters. $(C)$ The PTS targets the insulatorblocked IAB8 to the Tp promoter. Note the exclusive activation of $1 a c Z$ and strong facilitating effect (cf. W76 in $A$ ). (D) Deletion of $s u H w$ by the FLPFRT recombination system has no effect on promoter targeting as shown in the almost identical staining pattern as in $B$. $(E)$ Genomic PCR from three independent Flipped strains confirms that the insulator is, indeed, removed from the integrated transgene. $(F)$ The W98Flp strain shown in $D$ after transposition. The staining pattern indicates that promoter targeting is maintained in this strain (cf. $A, C)$.

function has not been reported for insulators. As we have demonstrated, an insulator is not necessary to maintain the promoter-targeting memory during transgene mobilization to new locations; thus, it is unlikely that insulator functions by directing the $P$-element to the right chromosomal location for integration. A third potential mechanism is that insulator may help recruit proteins that are necessary for PTS activity. This is also unlikely because we observed that promoter targeting only occurs when an insulator is inserted between an enhancer and its promoter (Q. Chen, Q. Lin, L. Lin, and J. Zhou, unpubl.). Because the insulator is a constitutive element that presumably recruits the same proteins regardless of its relative locations to the enhancer, or the promoter, the differential results on promoter targeting as a result of its location in the transgene is more likely due to which enhancer it blocks than what proteins it helps recruit for the PTS. We favor the model that the insulator is necessary for promoter targeting because of its domain boundary or enhancer-blocking function. For example, the PTS could recognize a special chromatin structure established by an insulator, such as specific histone or DNA modification, and then establish enhancer-promoter interactions over the insulator.

Two important implications could be gleaned from the role of an insulator in PTS function. First, the Fab insulator elements may play a role in $A b d-B$ that has not been previously realized. These elements are known to function as boundaries that keep individual regulatory domains functionally independent. The current study suggests that they are also necessary for long-range enhancer-promoter interactions in the $A b d-B$ locus, because without an insulator the PTS would not be able to target the distant, $A b d-B$ downstream enhancers such as IAB5 and IAB7 to their promoter, over long distances. Similar roles of insulators in long-range enhancer-promoter interactions have also been suggested by recent studies of the $s u H w$ insulator, where paired $s u H w$ could facilitate rather than block distantly located enhancers (Cai and Shen 2001; Muravyova et al. 2001). The second implication for the initial requirement of an insulator in promoter targeting is epigenetic inheritance. We observed that an insulator is required for promoter targeting, but only when the transgene is first generated. In established promoter-targeted lines, the insulator could be deleted without any adverse effect on promoter targeting. These results suggest that the PTS could epigenetically maintain promoter targeting through multiple generations (see below).

\section{The epigenetic inheritance of promoter targeting}

The most important implication of this study is the heritable nature of PTS function throughout the fly life cycle, which is transmittable into the next generations. This is supported by three sets of experiments presented here: enhancer cotargeting, the initial requirement of an insulator, and $P$-element transposition. Enhancer cotargeting strongly suggests that promoter targeting is a constitutive chromatin effect that is not determined at the onset of transcription, that it is independent of the function of an enhancer, and that it is epigenetically stable throughout the life cycle of a fly. Because promoter targeting is stable over all successive generations, the memory of the initial targeting event is likely epigenetically maintained by the PTS in the germ line. This idea is further supported by the differential requirement of insulators in the initiation and maintenance of promoter targeting. An insulator must be present to obtain transgenic strains that exhibit promoter targeting, but it could be deleted from the targeted strains, with no loss of promoter targeting. There are two alternative explanations for this result: Either the germ-line cells have the memory of promoter targeting, or the insulator must have helped the transgene to integrate into a chromosomal location where promoter targeting could occur.

More direct evidence for the epigenetic inheritance model comes from our subsequent $P$-element transposition experiment, which demonstrated that the promotertargeting effect is remarkably stable even when the transgene is translocated to new locations. In one of our experiments demonstrated in Figure 4, we found that when a $w$-targeted, W59-1 strain is transposed to various 
Lin et al.

locations, including two that moved to a different chromosome, enhancers from all new insertions appear to target the same $w$ promoter, suggesting that a memory is present within the transgene during its transposition to different locations in the germ-line cells. In a separate experiment shown in Figure 5, we generated new insertions from an original promoter-targeted strain, from which the suHw insulator has been removed by FLP-FRT recombination. Most of the new insertion strains (seven of nine) retain promoter targeting to the same $T p$ promoter. Although there are strain variations to the extent of how stable this memory is (Table 2C), these results suggest that an insulator is not required to maintain the promoter-targeting memory during transposition, and that an insulator probably does not function by directing the transgene to chromosome locations favorable for PTS function, as it is not present in the transgene when being transposed. Thus, in the original promoter-targeted strains, the consistent targeting to the same promoter generation after generation is largely due to epigenetic inheritance.

It could still be argued, however, that most $P$-element transpositions are relatively local where chromatin structures are similar, and hence the same type of promoter targeting can be generated without epigenetic memory. Considering that transpositions of nontargeted strains (Table 2A) are also local hops, yet produce a normal distribution of Type I, II, and III strains (see Fig. 1B), indistinguishable from generating promoter-targeted strains by DNA injection, the local chromatin near the original promoter-targeted transgene is less likely to exert a consistent effect on which promoter should be targeted when the $P$-element is excised and reinserted nearby. Presently, we could not rule out the possibility that a promoter-targeted transgene may associate with other chromatin structures or nuclear sites where similar function is processed. Consequently, such structure may dictate where the transgene could insert after being transposed. This possibility, however, is hinged on an epigenetic memory in the germ line, because some of the proteins associated with promoter targeting must be present to direct targeting after $P$-element reinsertion at a new site. We conclude that promoter targeting could be memorized in both somatic and germ-line cells and could be transmitted to successive generations.

Heritable transcription memory has been previously reported in the Drosophila $A b d-B$ locus. The $3.7-\mathrm{kb}$ $F a b-7$ boundary region also contains a cellular memory module (CMM) (Cavalli and Paro 1998; Bantignies et al. 2003) that can transmit a Polycomb-dependent heritable transcription memory to the offspring, which remains stable for a few generations. Similarly, the PTS-mediated promoter targeting appears to be stable in a majority of new insertions. However, once a newly targeting strain is obtained, it is stable in all successive generations, and can be transmitted through both the male and the female germ lines. It is not known what mechanism the PTS uses to transmit promoter targeting memory to subsequent generations. However, it is clearly different from what is used by the CMM, because PTS function is in- dependent of Polycomb (J. Zhou, unpubl.). Considering that certain chromatin loop structures could remain intact through spermatogenesis (Klaus et al. 2001), it is likely that the PTS function could be transmitted by a special loop structure (Fig. 1C, part b).

An important prediction of our study is that the inheritance of promoter targeting is not due to the transmission of an enhancer activity in the somatic cell into the germ line. It is a process that has to be determined in the germ-line lineage independent of enhancer functions (also in the absence of a history of enhancer activity) by a combination of the PTS, the promoter, the insulator, and, possibly, the local chromatin structure. The promoter-targeting activity detected in the embryo is not a de novo process, but it is built upon what has been determined in the germ-line cells. This prediction is supported by the FLP-FRT analysis and $P$-element transposition experiments because only germ-line recombination and transposition events could be recovered by our analysis. We propose that promoter-targeting memory is maintained by the PTS through either a stable chromatin loop or a special chromatin modification. Such structure or modification could be maintained in somatic tissues and germ-line cells in the absence of enhancer activity (Fig. 1C, parts a,b). When an enhancer becomes active, this structure or modification could automatically guide the enhancer to the targeted promoter (Fig. 1C, part c). This unique property of the PTS element underscores the possibility that certain aspects of the mechanism controlling long-range enhancer-promoter interactions in the Drosophila Hox cluster may be programmed in the germ-line chromatin.

\section{Materials and methods}

\section{Plasmid constructions}

The construction of the $P$-transformant vector $\mathrm{HN}$ was described in Zhou and Levine (1999). The construct HNP was made by inserting a BamHI-BglII fragment of the 625-bp PTS into the BglII site of HN. Similarly, W59 was made by inserting a 1.7-kb DNA that contains both the PTS and the Fab-8 insulator. W89 and W91 were made by inserting the 1.6-kb PstI fragment of IAB8, or the 1.0-kb PstI IAB5 DNA into the PstI site of CaSperN vector (Q. Lin and J. Zhou, unpubl.). W71 and W72 were made by inserting a 380-bp fragment of $s u H w$ into the NotI site of W91 and W89, respectively. W93 and W83 were created by inserting the 625-bp PTS into the NotI site of these vectors. To generate the $P$-transgene W34, a 700-bp BamHI-BglII fragment containing the 625-bp PTS and part of the Bluescript polylinker, as well as a 1.6-kb BamHI-BglII IAB8 was first cloned into the BglII site of C4PLZ. Then, the 800-bp BglII fragment containing FRT and Fab-8 was inserted into the recreated BglII site (the 580-bp BamHI fragment of Fab-8 was first inserted into a BamHI site located between a direct repeat of FRT sites in Bluescript). W97, W98, W87, and W88 were made by inserting an NotI fragment that contains the 625-bp PTS and FRT sites flanking suHw into W89 and W91, respectively. This NotI fragment was made by first inserting the BamHI-BglII PTS fragment into the BamHI site, followed by adding the FRT-flanked suHw fragment (made by inserting the BamHI suHw between the two FRT sites in Bluescript) into the BamHI site. 
W123 was made by inserting a 300-bp NEE enhancer between the $w$ and $T p$ promoters in the transgene shown in Figure 5 of Zhou and Levine (1999). To make W177 and W179, the BamHIBglII fragment of the lacZ gene from the C4PLZ vector was replaced by the BamHI-BglII fragment of Tp-GFP. A BamHI and BglII fragment was later inserted. The BglII site was then converted into an NotI site, into which an assortment of elements including suHw, PTS, glass, and IAB5 (W177) or IAB8 (W179) was inserted. These DNA fragments were constructed in Bluescript that contains two NotI sites. To make W182, W191, W198, and W199, the C4PLZ vector was similarly modified as above, but only the eve-GFP gene was inserted. At the 3 '-end of GFP, four combinations of different DNA fragments were inserted. For W191, they are suHw, PTS, glass, NEE, and IAB5; for W182, these are Fab-8, PTS, glass, NEE, and IAB5; for W199, these elements are $s u H w$, PTS, glass, HI, and IAB5; and finally for W198, Fab-8, PTS, glass, HI, and IAB5.

\section{P-transformation and in situ hybridization}

P-element transformation vectors containing lacZ and white reporter genes were introduced into the Drosophila germ line by injecting $y w^{67}$ embryos as described previously (Rubin and Spradling 1982). Approximately 20-30 independent transformants were obtained for each of the recombinant $P$-elements shown. In situ hybridizations were performed essentially as described in previous reports (Tautz and Pfeifle 1989; Zhou et al. 1999).

\section{Fly strains and crosses}

Transgenic flies expressing the Flip recombinase were kindly provided by Gary Struhl (Department of Genetics and Development, Howard Hughes Medical Institute, Columbia University, New York) and Steve Small (Biology Department, New York University, New York) (Wu et al. 1998). To recombine different DNA elements away from the transgenes, females carrying the transgene (provided that the transgene were located on the second or third chromosome) are mated with males that carry a $P$-transformation vector that expresses the Flp recombinase under the control of a sperm-specific tubulin promoter (Wu et al. 1998). The recombinase binds the FRT sites and deletes the intervening DNA. Male flies were collected and mated to yw virgin females to establish stocks that are subsequently analyzed by in situ hybridization.

\section{P-element mobilization}

To create new insertion sites for the $P$-elements, flies carrying the $P$-elements were mated with flies expressing the $\Delta 2-3$ transposase, en mass. Female $F_{1}$ flies with both the $P$-element and the transposase were mated with yw males. Male offspring bearing darker eye colors were collected and used to establish individual lines. Each new insertion line was then mapped. $P$-elements that hopped to different chromosomes were segregated from the original $P$-elements and kept as stocks.

\section{Genomic DNA preparations and PCR analysis}

Genomic DNA preparation and PCR was adopted from BDGP (http://www.fruitfly.org/about/methods/index.html). Briefly, five anesthetized flies were collected in Eppendorf tubes and frozen at $-20^{\circ} \mathrm{C}$, then were homogenized in $100 \mu \mathrm{L}$ of buffer $\mathrm{A}$ (100 mM Tris- $\mathrm{HCl}$ at $\mathrm{pH}$ 7.5, $100 \mathrm{mM}$ EDTA, $100 \mathrm{mM} \mathrm{NaCl}$, $0.5 \%$ SDS) with a disposable tissue grinder. After incubation for $20 \mathrm{~min}$ at $65^{\circ} \mathrm{C}, 200 \mu \mathrm{L}$ of $\mathrm{LiCl} / \mathrm{Kac}(1$ part $5 \mathrm{M} \mathrm{KAc}: 2.5$ parts 6 $\mathrm{M} \mathrm{LiCl}$ ) was added to the tube and incubated for $10 \mathrm{~min}$ on ice. The mixture was spun for $15 \mathrm{~min}$, and the supernatant was transferred to a fresh tube, followed by precipitation with 150 $\mu \mathrm{L}$ of iso-propanol. The DNA pellet was washed with $70 \%$ ethanol, air-dried, and resolved in $50 \mu \mathrm{L}$ of TE. Two microliters of the DNA sample was used in genomic PCR with primers specific in the PTS and enhancer regions that flank the SuHw insulator. Ten percent of the PCR product was loaded on a $1 \%$ agarose gel.

\section{Acknowledgments}

We thank Jame Jaynes, Alex Mazo, and Sheryl Smith for critically reading the manuscript; and Donald Rio and Keith Magert for insightful discussions. This work is supported by NIH GM65391, the March of Dimes Birth Defect Foundation, the Edward Mallinckrodt Jr. Foundation, and the Concern Foundation to J.Z. This work is also partially supported by funds from the Commonwealth Universal Research Enhancement Program, Pennsylvania Department of Health.

\section{References}

Bantignies, F., Grimaud, C., Lavrov, S., Gabut, M., and Cavalli, G. 2003. Inheritance of Polycomb-dependent chromosomal interactions in Drosophila. Genes \& Dev. 17: 2406-2420.

Barges, S., Mihaly, J., Galloni, M., Hagstrom, K., Muller, M., Shanower, G., Schedl, P., Gyurkovics, H., and Karch, F. 2000. The Fab-8 boundary defines the distal limit of the bithorax complex iab-7 domain and insulates iab-7 from initiation elements and a PRE in the adjacent iab-8 domain. Development 127: 779-790.

Bell, A.C. and Felsenfeld, G. 1999. Stopped at the border: Boundaries and insulators. Curr. Opin. Genet. Dev. 9: 191-198.

- 2000. Methylation of a CTCF-dependent boundary controls imprinted expression of the Igf2 gene. Nature 405: 482485.

Bell, A.C., West, A.G., and Felsenfeld, G. 1999. The protein CTCF is required for the enhancer blocking activity of vertebrate insulators. Cell 98: 387-396.

- 2001. Insulators and boundaries: Versatile regulatory elements in the eukaryotic genome. Science 291: 447-450.

Bender, W. and Hudson, A. 2000. P element homing to the Drosophila bithorax complex. Development 127: 3981-3992.

Busturia, A. and Bienz, M. 1993. Silencers in abdominal-B, a homeotic Drosophila gene. EMBO J. 12: 1415-1425.

Cai, H. and Levine, M. 1995. Modulation of enhancer-promoter interactions by insulators in the Drosophila embryo. Nature 376: 533-536.

Cai, H.N. and Shen, P. 2001. Effects of cis arrangement of chromatin insulators on enhancer-blocking activity. Science 291: 493-495.

Cavalli, G. and Paro, R. 1998. The Drosophila Fab-7 chromosomal element conveys epigenetic inheritance during mitosis and meiosis. Cell 93: 505-518.

Celniker, S.E., Sharma, S., Keelan, D.J., and Lewis, E.B. 1990. The molecular genetics of the bithorax complex of Drosophila: Cis-regulation in the Abdominal-B domain. EMBO J. 9: 4277-4286.

Chung, J.H., Whiteley, M., and Felsenfeld, G. 1993. A 5' element of the chicken $\beta$-globin domain serves as an insulator in human erythroid cells and protects against position effect in Drosophila. Cell 74: 505-514.

Dorsett, D. 1993. Distance-independent inactivation of an enhancer by the suppressor of Hairy-wing DNA-binding protein of Drosophila. Genetics 134: 1135-1144.

Duncan, I. 1987. The bithorax complex. Annu. Rev. Genet. 21: 285-319. 
Galloni, M., Gyurkovics, H., Schedl, P., and Karch, F. 1993. The bluetail transposon: Evidence for independent cis-regulatory domains and domain boundaries in the bithorax complex. EMBO J. 12: 1087-1097.

Gaszner, M., Vazquez, J., and Schedl, P. 1999. The Zw5 protein, a component of the scs chromatin domain boundary, is able to block enhancer-promoter interaction. Genes \& Dev. 13: $2098-2107$.

Gerasimova, T.I., Gdula, D.A., Gerasimov, D.V., Simonova, O., and Corces, V.G. 1995. A Drosophila protein that imparts directionality on a chromatin insulator is an enhancer of position-effect variegation. Cell 82: 587-597.

Gerasimova, T.I., Byrd, K., and Corces, V.G. 2000. A chromatin insulator determines the nuclear localization of DNA. Mol. Cell 6: 1025-1035.

Geyer, P.K. and Corces, V.G. 1992. DNA position-specific repression of transcription by a Drosophila zinc finger protein. Genes \& Dev. 6: 1865-1873.

Golic, K.G. and Lindquist, S. 1989. The FLP recombinase of yeast catalyzes site-specific recombination in the Drosophila genome. Cell 59: 499-509.

Grewal, S.I. and Elgin, S.C. 2002. Heterochromatin: New possibilities for the inheritance of structure. Curr. Opin. Genet. Dev. 12: 178-187.

Gyurkovics, H., Gausz, J., Kummer, J., and Karch, F. 1990. A new homeotic mutation in the Drosophila bithorax complex removes a boundary separating two domains of regulation. EMBO I. 9: 2579-2585.

Hagstrom, K. and Schedl, P. 1997. Remembrance of things past: Maintaining gene expression patterns with altered chromatin. Curr. Opin. Genet. Dev. 7: 814-821.

Hagstrom, K., Muller, M., and Schedl, P. 1996. Fab-7 functions as a chromatin domain boundary to ensure proper segment specification by the Drosophila bithorax complex. Genes \& Dev. 10: 3202-3215.

Hall, I.M., Shankaranarayana, G.D., Noma, K., Ayoub, N., Cohen, A., and Grewal, S.I. 2002. Establishment and maintenance of a heterochromatin domain. Science 297: 2232-2237.

Hark, A.T., Schoenherr, C.J., Katz, D.J., Ingram, R.S., Levorse, J.M., and Tilghman, S.M. 2000. CTCF mediates methylation-sensitive enhancer-blocking activity at the H19/Igf2 locus. Nature 405: 486-489.

Hendrickson, J.E. and Sakonju, S. 1995. Cis and trans interactions between the iab regulatory regions and abdominal-A and abdominal-B in Drosophila melanogaster. Genetics 139: $835-848$.

Hopmann, R., Duncan, D., and Duncan, I. 1995. Transvection in the iab-5,6,7 region of the bithorax complex of Drosophila: Homology independent interactions in trans. Genetics 139: 815-833.

Ip, Y.T., Park, R.E., Kosman, D., Bier, E., and Levine, M. 1992. The dorsal gradient morphogen regulates stripes of rhomboid expression in the presumptive neuroectoderm of the Drosophila embryo. Genes \& Dev. 6: 1728-1739.

Ishii, K., Arib, G., Lin, C., Van Houwe, G., and Laemmli, U.K. 2002. Chromatin boundaries in budding yeast: The nuclear pore connection. Cell 109: 551-562.

Kanduri, C., Pant, V., Loukinov, D., Pugacheva, E., Qi, C.F., Wolffe, A., Ohlsson, R., and Lobanenkov, V.V. 2000. Functional association of CTCF with the insulator upstream of the $\mathrm{H} 19$ gene is parent of origin-specific and methylationsensitive. Curr. Biol. 10: 853-856.

Kassis, J.A. 2002. Pairing-sensitive silencing, polycomb group response elements, and transposon homing in Drosophila. Adv. Genet. 46: 421-438.

Kellum, R. and Schedl, P. 1992. A group of scs elements func- tion as domain boundaries in an enhancer-blocking assay. Mol. Cell. Biol. 12: 2424-2431.

Kennison, J.A. 1995. The Polycomb and Trithorax group proteins of Drosophila: Trans-regulators of homeotic gene function. Annu. Rev. Genet. 29: 289-303.

Klaus, A.V., McCarrey, J.R., Farkas, A., and Ward, W.S. 2001. Changes in DNA loop domain structure during spermatogenesis and embryogenesis in the Syrian golden hamster. Biol. Reprod. 64: 1297-1306.

Lin, Q., Wu, D., and Zhou, J. 2003. The promoter targeting sequence facilitates and restricts a distant enhancer to a single promoter in the Drosophila embryo. Development 130: $519-526$.

Mallin, D.R., Myung, J.S., Patton, J.S., and Geyer, P.K. 1998. Polycomb group repression is blocked by the Drosophila suppressor of Hairy-wing $[\mathrm{su}(\mathrm{Hw})]$ insulator. Genetics 148: 331-339.

Mihaly, J., Hogga, I., Gausz, J., Gyurkovics, H., and Karch, F. 1997. In situ dissection of the Fab-7 region of the bithorax complex into a chromatin domain boundary and a Polycomb-response element. Development 124: 1809-1820.

Mihaly, J., Hogga, I., Barges, S., Galloni, M., Mishra, R.K., Hagstrom, K., Muller, M., Schedl, P., Sipos, L., Gausz, J., et al. 1998. Chromatin domain boundaries in the Bithorax complex. Cell Mol. Life Sci. 54: 60-70.

Morgan, H.D., Sutherland, H.G., Martin, D.I., and Whitelaw, E. 1999. Epigenetic inheritance at the agouti locus in the mouse. Nat. Genet. 23: 314-318.

Moses, K. and Rubin, G.M. 1991. Glass encodes a site-specific DNA-binding protein that is regulated in response to positional signals in the developing Drosophila eye. Genes \& Dev. 5: 583-593.

Muravyova, E., Golovnin, A., Gracheva, E., Parshikov, A., Belenkaya, T., Pirrotta, V., and Georgiev, P. 2001. Loss of insulator activity by paired $\mathrm{Su}(\mathrm{Hw})$ chromatin insulators. Science 291: 495-498.

Nakayama, J., Klar, A.J., and Grewal, S.I. 2000. A chromodomain protein, Swi6, performs imprinting functions in fission yeast during mitosis and meiosis. Cell 101: 307-317.

Noma, K., Allis, C.D., and Grewal, S.I. 2001. Transitions in distinct histone $\mathrm{H} 3$ methylation patterns at the heterochromatin domain boundaries. Science 293: 1150-1155.

Pirrotta, V. 1998. Polycombing the genome: PcG, trxG, and chromatin silencing. Cell 93: 333-336.

Prioleau, M.N., Nony, P., Simpson, M., and Felsenfeld, G. 1999. An insulator element and condensed chromatin region separate the chicken $\beta$-globin locus from an independently regulated erythroid-specific folate receptor gene. EMBO $J$. 18: 4035-4048.

Rakyan, V. and Whitelaw, E. 2003. Transgenerational epigenetic inheritance. Curr. Biol. 13: R6.

Rakyan, V.K., Preis, J., Morgan, H.D., and Whitelaw, E. 2001. The marks, mechanisms and memory of epigenetic states in mammals. Biochem. J. 356: 1-10.

Rakyan, V.K., Chong, S., Champ, M.E., Cuthbert, P.C., Morgan, H.D., Luu, K.V., and Whitelaw, E. 2003. Transgenerational inheritance of epigenetic states at the murine Axin $(\mathrm{Fu})$ allele occurs after maternal and paternal transmission. Proc. Nat1. Acad. Sci. 100: 2538-2543.

Riddihough, G. and Ish-Horowicz, D. 1991. Individual stripe regulatory elements in the Drosophila hairy promoter respond to maternal, gap, and pair-rule genes. Genes \& Dev. 5: 840-854.

Rubin, G.M. and Spradling, A.C. 1982. Genetic transformation of Drosophila with transposable element vectors. Science 218: $348-353$. 
Scott, K.S. and Geyer, P.K. 1995. Effects of the su(Hw) insulator protein on the expression of the divergently transcribed Drosophila yolk protein genes. EMBO J. 14: 6258-6267.

Sigrist, C.J. and Pirrotta, V. 1997. Chromatin insulator elements block the silencing of a target gene by the Drosophila polycomb response element (PRE) but allow trans interactions between PREs on different chromosomes. Genetics 147: 209-221.

Simon, J. 1995. Locking in stable states of gene expression: Transcriptional control during Drosophila development. Curr. Opin. Cell Biol. 7: 376-385.

Sipos, L., Mihaly, J., Karch, F., Schedl, P., Gausz, J., and Gyurkovics, H. 1998. Transvection in the Drosophila Abd-B domain: Extensive upstream sequences are involved in anchoring distant cis-regulatory regions to the promoter. Genetics 149: 1031-1050.

Small, S., Arnosti, D.N., and Levine, M. 1993. Spacing ensures autonomous expression of different stripe enhancers in the even-skipped promoter. Development 119: 762-772.

Tautz, D. and Pfeifle, C. 1989. A non-radioactive in situ hybridization method for the localization of specific RNAs in Drosophila embryos reveals translational control of the segmentation gene hunchback. Chromosoma 98: 81-85.

Wu, X., Vakani, R., and Small, S. 1998. Two distinct mechanisms for differential positioning of gene expression borders involving the Drosophila gap protein giant. Development 125: 3765-3774.

Yusufzai, T.M., Tagami, H., Nakatani, Y., and Felsenfeld, G. 2004. CTCF tethers an insulator to subnuclear sites, suggesting shared insulator mechanisms across species. Mol. Cell 13: 291-298.

Zhao, K., Hart, C.M., and Laemmli, U.K. 1995. Visualization of chromosomal domains with boundary element-associated factor BEAF-32. Cell 81: 879-889.

Zhou, J. and Levine, M. 1999. A novel cis-regulatory element, the PTS, mediates an anti-insulator activity in the Drosophila embryo. Cell 99: 567-575.

Zhou, J., Barolo, S., Szymanski, P., and Levine, M. 1996. The Fab-7 element of the bithorax complex attenuates enhancerpromoter interactions in the Drosophila embryo. Genes \& Dev. 10: 3195-3201.

Zhou, J., Ashe, H., Burks, C., and Levine, M. 1999. Characterization of the transvection mediating region of the abdominal-B locus in Drosophila. Development 126: 3057-3065. 


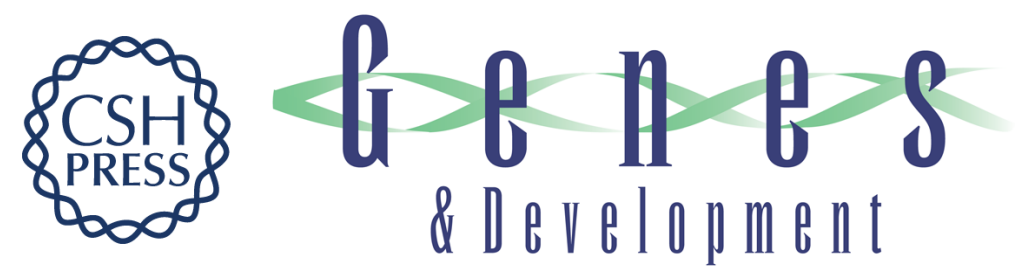

\title{
The Promoter Targeting Sequence mediates epigenetically heritable transcription memory
}

\author{
Qing Lin, Qi Chen, Lan Lin, et al.
}

Genes Dev. 2004, 18:

Access the most recent version at doi:10.1101/gad.1230004

$\begin{array}{ll}\text { References } & \begin{array}{l}\text { This article cites } 63 \text { articles, } 29 \text { of which can be accessed free at: } \\ \text { http://genesdev.cshlp.org/content/18/21/2639.full.html\#ref-list-1 }\end{array}\end{array}$

License Email Alerting
Service $\begin{aligned} & \text { Receive free email alerts when new articles cite this article - sign up in the box at the top } \\ & \text { right corner of the article or click here. }\end{aligned}$

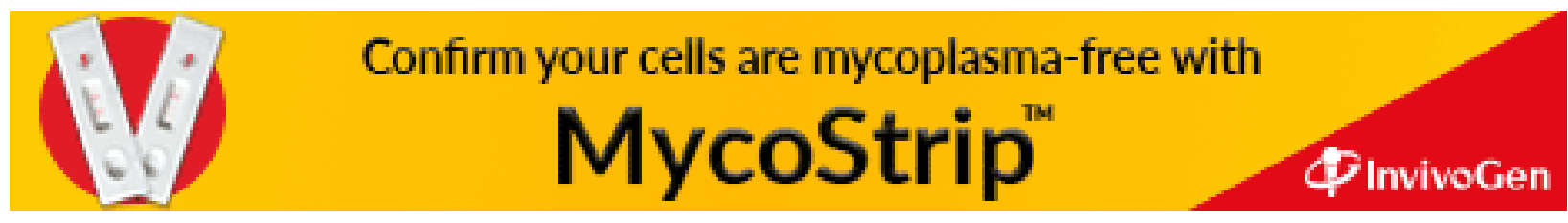

